\title{
Anaphylaxis in the Avascular Tissue of the Rabbit Cornea
} A.C. Breebaart

From the Ophthalmic Hospital (Prof. Dr. A. Hagedoorn) and the Laboratory of Histology (Prof. Dr. G. C. Heringa) of the University of Amsterdam

Report of a new phenomenon of corneal anaphylaxis (so-called ring reaction), in contrast to the phenomena known thus far not accompanied by a dense ingrowth of vessels. 